\title{
Subset sums avoiding quadratic nonresidues
}

\author{
by \\ PÉTER Csikvári (Budapest)
}

1. Introduction. It is a well-known problem to estimate the largest clique of the Paley graph, i.e., to estimate $|A|$ for $A \subset F_{p}(p \equiv 1(\bmod 4))$ such that $A-A=\left\{a-a^{\prime} \mid a, a^{\prime} \in A\right\}$ avoids the set of quadratic nonresidues. In this paper we study a much simpler problem, namely with $A-A$ replaced by the set

$$
\operatorname{FS}(A)=\left\{\sum \varepsilon_{a} a \mid \varepsilon_{a}=0 \text { or } 1 \text { and } \sum \varepsilon_{a}>0\right\} .
$$

In other words, we will estimate the maximal cardinality of $A \subset F_{p}$ such that $\mathrm{FS}(A)$ avoids the set of quadratic nonresidues. We show that this problem is strongly related to the problem of estimating the least quadratic nonresidue $n(p)$, since the set $\left\{1,2, \ldots,\left[n(p)^{1 / 2}\right]\right\}$ satisfies the above condition. We prove that the maximal value of $|A|$ is $\Omega(\log \log p)$. On the other hand, we show that $|A|=O\left(n(p) \log ^{3} p\right)$. The proof is based on the fact that if $t$ is a quadratic nonresidue then $\operatorname{FS}(A) \cap t \cdot \operatorname{FS}(A)=\emptyset$ or $\{0\}$ where by definition $t \cdot B=\{t b \mid b \in B\}$. We show that if $t$ is small then $|\mathrm{FS}(A)|$ is much greater than $|A|$.

In the next section we study the case when $t=n(p)=2$. In Section 3 we prove the upper bound $|A|=O\left(n(p) \log ^{3} p\right)$. In the last section we show that the maximal value of $|A|$ is $\Omega(\log \log p)$.

2. The case $n(p)=2$. In the case $n(p)=2$ we have $\operatorname{FS}(A) \cap 2 \cdot \operatorname{FS}(A)$ $=\emptyset$ or $\{0\}$. First we consider the case $\operatorname{FS}(A) \cap 2 \cdot \operatorname{FS}(A)=\emptyset$.

Theorem 2.1. If $\operatorname{FS}(A) \cap 2 \cdot \operatorname{FS}(A)=\emptyset$ then $|\operatorname{FS}(A)|=2^{|A|}$.

Proof. We have to show that if $\operatorname{FS}(A) \cap 2 \cdot \operatorname{FS}(A)=\emptyset$ then all the subset sums are different. Indeed, if two different sums had the same value then omitting the intersection we would get $s=a_{i_{1}}+\cdots+a_{i_{l}}=a_{j_{1}}+\cdots+a_{j_{m}}$

2000 Mathematics Subject Classification: Primary 11B75.

Key words and phrases: subset sums, quadratic residues. 
$\left(i_{u} \neq j_{v}\right)$. In this case $s$ and $2 s=a_{i_{1}}+\cdots+a_{i_{l}}+a_{j_{1}}+\cdots+a_{j_{m}}$ would be in $\operatorname{FS}(A)$, which contradicts the assumption.

A trivial consequence of Theorem 2.1 is

Corollary 2.2. If $n(p)=2$ (i.e. $\left(\frac{2}{p}\right)=-1$ ) and every element of $\mathrm{FS}(A)$ is a quadratic residue then $|A| \leq(\log p) / \log 2$.

Theorem 2.3. Assume that $0 \notin A$. If $\mathrm{FS}(A) \cap 2 \cdot \mathrm{FS}(A)=\emptyset$ or $\{0\}$ then $|A| \leq(2 \log p) / \log 2$.

REMARK $1.0 \notin A$ is just a simplifying condition: if we leave out the 0 from $A$ then $\operatorname{FS}(A)$ will not change and the cardinality of $A$ will only decrease by 1 .

Proof of Theorem 2.3. We will say that $\sum_{i \in I} a_{i}=a$ is an irreducible $a$ sum if there is no $\emptyset \neq J \subset I$ for which $\sum_{i \in J} a_{i}=0$. Two irreducible $a$-sums are disjoint, because if $\sum_{i \in I_{1}} a_{i}=\sum_{j \in I_{2}} a_{j}$ then $\sum_{i \in I_{1} \backslash I_{2}} a_{i}=\sum_{i \in I_{2} \backslash I_{1}} a_{i}=$ $s \neq 0$ and $s, 2 s \in \mathrm{FS}(A)$ contradicts the assumption. On the other hand, in case $a \neq 0$ there cannot be two disjoint irreducible $a$-sums. Thus we only get an $a$-sum as the sum of "the" irreducible $a$-sum and a 0 -sum. Each 0 -sum is a sum of irreducible 0 -sums so the number of 0 -sums is at most $2^{|A| / 2}$ since every irreducible 0 -sum has at least two elements (here we have used the simplifying condition that $0 \notin A$ ). Hence $p \cdot 2^{|A| / 2} \geq 2^{|A|}$, which yields the conclusion.

Corollary 2.4. If $n(p)=2$ and every element of $\mathrm{FS}(A)$ is a square mod $p$ then $|A| \leq(2 \log p) / \log 2$.

Corollary 2.5. If $A \subset\{1, \ldots, N\}$ and every element of $\operatorname{FS}(A)$ is a perfect square then $|A|=O(\log \log N)$.

Proof. We will use Gallagher's larger sieve [4]. Let $y=40 \log N \log \log N$ and let $S=\{p \leq y \mid p$ prime, $p \equiv 3$ or $5(\bmod 8)\}$. By Corollary 2.4, $\nu(p) \leq(2 \log p) / \log 2$ for these primes $p$. By the larger sieve

$$
|A| \leq \frac{\sum_{p \in S} \Lambda(p)-\log N}{\sum_{p \in S} \frac{\Lambda(p)}{\nu(p)}-\log N}
$$

if the denominator is positive. We have

$$
\log y \leq 2 \log \log N
$$

if $N$ is large enough. Furthermore

$$
\sum_{p \in S} \Lambda(p)=\frac{1}{2} y+o(y)
$$


and

$$
\sum_{p \in S} \frac{\Lambda(p)}{\nu(p)} \geq \frac{(\log 2) y}{4 \log y}+o\left(\frac{y}{\log y}\right) \geq \frac{y}{10 \log y}
$$

if $y$, thus also $N$, is large enough. Hence for large $N$,

$$
\sum_{p \in S} \frac{\Lambda(p)}{\nu(p)} \geq \frac{40 \log N \log \log N}{20 \log \log N}=2 \log N .
$$

Thus $|A| \leq 40 \log \log N$.

3. Upper bound. First we will prove a theorem on Abelian groups from which the upper bound follows.

Theorem 3.1. Let $A \subset G$ where $G$ is a finite Abelian group. Assume that $|A| \geq 2000 t \log ^{3}|G|$. Then there exists a $d \neq 0$ for which $\{d, 2 d, \ldots, t d\}$ $\subset \mathrm{FS}(A)$.

Proof. We argue by contradiction. Assume that there exists a set $A$ for which $|A|=n>2000 t \log ^{3}|G|$ such that $\mathrm{FS}(A)$ does not contain any set $\{d, 2 d, \ldots, t d\}$ where $d \neq 0$. We can also assume that $0 \notin A$. Let $r$ be a fixed positive integer which we will choose later. We will use the Erdös-Rado theorem on $\Delta$-systems.

Lemma 3.2 (Erdös-Rado). Assume that $A_{1}, \ldots, A_{m}$ are subsets of a given set such that $m \geq r !(t-1)^{r}$ and $\left|A_{i}\right|=r$. Then they contain a $\Delta$ system with $t$ elements, i.e., $A_{i_{1}}, \ldots, A_{i_{t}}, i_{1}<\cdots<i_{t}$, such that $A_{i_{k}} \cap A_{i_{l}}=$ $\bigcap_{j=1}^{t} A_{t_{j}}$ for all $1 \leq k<l \leq t$.

Again we first give an upper bound for the number of irreducible sums. (We recall that $\sum_{a \in I} a$ is irreducible if there is no $J \neq \emptyset$ with $J \subset I$ such that $\sum_{a \in J} a=0$, and we call a sum an irreducible $a$-sum if it is irreducible and its value is $a$.) We estimate the number of $r$-term irreducible $a$-sums. If $a \neq 0$ then there exist at most $r !(t-1)^{r} r$-term irreducible $a$-sums. Indeed, otherwise these sums as a set contain a $\Delta$-system with $t$ elements by the lemma. If we leave out the intersection of the sums of sets we get $t$ disjoint sums having the same nonzero value since the sums were irreducible. Let $d$ be the value of these sums. Then adding together some of these disjoint sums we find that $\{d, 2 d, \ldots, t d\} \subset \mathrm{FS}(A)$ contradicting the assumption. This argument cannot be applied for $a=0$ immediately since it may occur that $t$ disjoint irreducible $r$-term sums form a $\Delta$-system. Although we can easily solve this problem, now we can say that there are at most $n(r-1) !(t-1)^{r-1}$ irreducible 0 -sums since if there are more then there is an $a \in A$ appearing in more than $(r-1) !(t-1)^{r-1}$ irreducible sums as a summand. Omitting $a$ from these sums we get the previous case with $(r-1)$-term sums instead 
of $r$, since these new sums have value $-a$ which is not 0 as $0 \notin A$, and are irreducible since a subsum of an irreducible sum is still irreducible.

Now we give an upper bound for the number of $r$-term $a$-sums. Every $a$-sum is a sum of an irreducible $a$-sum and some irreducible 0 -sums (this decomposition is, of course, not unique, but this is not a problem since we only give an upper bound). Let us consider those representations where the irreducible $r$-term $a$-sum has $k_{1}$ terms and the irreducible 0 -sums have $k_{2}, \ldots, k_{m}$ terms, respectively. According to the previous argument the number of these sums is at most

$$
\begin{aligned}
k_{1} !(t-1)^{k_{1}} n\left(k_{2}-1\right) !(t-1)^{k_{2}-1} \cdots n\left(k_{m}-1\right) !(t-1)^{k_{m}-1} & \\
& \leq \prod_{i=1}^{m}\left(n\left(k_{i}-1\right) !(t-1)^{k_{i}-1}\right)=n^{m}\left(\prod_{i=1}^{m}\left(k_{i}-1\right) !\right)(t-1)^{r-m},
\end{aligned}
$$

since $\sum_{i=1}^{m} k_{i}=r$ and we will choose $r$ later so that $k_{1}(t-1) \leq r(t-1) \leq n$. We now show that

$$
n^{m}\left(\prod_{i=1}^{m}\left(k_{i}-1\right) !\right)(t-1)^{r-m} \leq r^{r / 2} n^{r / 2+1}(t-1)^{r / 2} .
$$

Indeed, since every irreducible 0 -sum has at least two elements (as $0 \notin A$ ), we have $m-1 \leq r / 2$ and $n^{r / 2+1-m} \geq(r(t-1))^{r / 2+1-m}$. Hence

$$
\begin{aligned}
r^{r / 2} n^{r / 2+1}(t-1)^{r / 2} & \geq r^{r / 2} n^{m}(r(t-1))^{r / 2+1-m}(t-1)^{r / 2} \\
& \geq n^{m} r^{r-m}(t-1)^{r-m} \geq n^{m}\left(\prod_{i=1}^{m}\left(k_{i}-1\right) !\right)(t-1)^{r-m},
\end{aligned}
$$

since $\prod_{i=1}^{m}\left(k_{i}-1\right) ! \leq(r-m) ! \leq r^{r-m}$. Let $p(r)$ denote the number of partitions of $r$. Then every $a \in G$ can be represented as a sum of $r$ elements of $A$ in at most $p(r) r^{r / 2} n^{r / 2+1}(t-1)^{r / 2}$ ways. Since there are $\left(\begin{array}{l}n \\ r\end{array}\right) r$-term sums we have

$$
\left(\begin{array}{l}
n \\
r
\end{array}\right) \leq|G| \cdot p(r) r^{r / 2} n^{r / 2+1}(t-1)^{r / 2} .
$$

We will choose $r$ so that

$$
\frac{\left(\begin{array}{l}
n \\
r
\end{array}\right)}{p(r) r^{r / 2} n^{r / 2+1}(t-1)^{r / 2}}
$$

is nearly maximal. For two consecutive $r$ 's consider the fraction

$$
\begin{aligned}
\frac{\left(\begin{array}{l}
n \\
r
\end{array}\right)}{p(r) r^{r / 2} n^{r / 2+1}(t-1)^{r / 2}}: & \frac{\left(\begin{array}{c}
n \\
r+1
\end{array}\right)}{p(r+1)(r+1)^{(r+1) / 2} n^{(r+1) / 2+1}(t-1)^{(r+1) / 2}} \\
& =\frac{r+1}{n-r} \frac{p(r+1)}{p(r)}\left(1+\frac{1}{r}\right)^{r / 2}(n(r+1)(t-1))^{1 / 2} .
\end{aligned}
$$

For the best choice of $r$ this must be approximately 1. Let us choose $r=$ 
$\left[n^{1 / 3}: e(t-1)^{1 / 3}\right]$; up to a constant factor this is the best choice. Now we can use the elementary estimates $m(m / e)^{m}>m !>(m / e)^{m}$ for $m \geq 6$ to obtain

$$
\begin{aligned}
|G| & \geq \frac{\left(\begin{array}{l}
n \\
r
\end{array}\right)}{p(r) r^{r / 2} n^{r / 2+1}(t-1)^{r / 2}} \\
& \geq \frac{\left(\frac{n}{e}\right)^{n}}{r(n-r)\left(\frac{r}{e}\right)^{r}\left(\frac{n-r}{e}\right)^{n-r} p(r) r^{r / 2} n^{r / 2+1}(t-1)^{r / 2}} \\
& =\frac{1}{n r(n-r) p(r)}\left(\frac{n}{n-r}\right)^{n-r}\left(\frac{n^{1 / 2}}{r^{3 / 2}(t-1)^{1 / 2}}\right)^{r} \geq \frac{1}{|G|^{3} p(r)}\left(e^{3 / 2}\right)^{r} .
\end{aligned}
$$

In the latter inequality we have used the fact that $|G| \geq \max \{n, r, n-r\}$. Now we use the classical estimate

$$
p(r)<\exp \left(\frac{2 \pi}{\sqrt{6}} \sqrt{r}\right)<\exp \left(\frac{1}{2} r\right) .
$$

It follows that $|G|^{4}>e^{r}$ so $4 \log |G| \geq r$. Thus $4^{3} \log ^{3}|G| \geq r^{3}>n / 30(t-1)$, whence $2000(t-1) \log ^{3}|G|>n$, contrary to assumption.

REMARK 2. The basic idea of this proof comes from an article of Erdös and Sárközy [3], who study what can be said about the length of an arithmetic progression contained in the set of subset sums of a subset of $\{1, \ldots, N\}$.

The statement of Theorem 3.1 is nearly sharp since for the set

$$
A=\{t, t+1, \ldots,[\sqrt{2} t]\} \subset Z_{n}
$$

with $t^{3}<n$ no two elements of $\operatorname{FS}(A)$ have quotient $t$, and $|A|=\Omega(t)$. On the other hand, a basis of $Z_{3}^{n}$ shows that the set of subset sums does not contain two elements with quotient 2 , and we have $|A|=\Omega\left(\log \left|Z_{3}\right|^{n}\right)$. Other much trickier examples can be found in the above mentioned article.

Corollary 3.3. Let $A \subset F_{p}$. Assume that $\mathrm{FS}(A)$ avoids the quadratic nonresidues. Then $|A|=O\left(n(p) \log ^{3} p\right)$, where $n(p)$ denotes the least quadratic nonresidue.

Proof. Otherwise one can apply Theorem 3.1 with $t=n(p)$ to deduce that there exists a $d \neq 0$ for which $d$ and $n(p) d$ are both quadratic residues, which is a contradiction.

REMARK 3. If we also assume that $0 \notin \mathrm{FS}(A)$, i.e., every element of $\mathrm{FS}(A)$ is a quadratic residue, then $|A|=O\left(n(p) \log ^{2} p\right)$, so that we can win a factor $\log p$ since we do not need to estimate the number of irreducible sums, and we can apply the Erdös-Rado theorem immediately. On the other hand, obviously one can replace the set of quadratic nonresidues by the set of quadratic residues, since one can multiply each element of $A$ with the same quadratic nonresidue and by construction no subset sum of the new set is a quadratic residue. 
REMARK 4. Since $n(p)=O_{\varepsilon}\left(p^{1 / 4 \sqrt{e}+\varepsilon}\right)$ [1], we get this upper bound also for the maximal value of $|A|$. According to a result of Burgess and Elliott [2], if $g(p)$ denotes the least primitive root modulo $p$ then

$$
\frac{1}{\pi(x)} \sum_{p \leq x} g(p) \leq C \log ^{2} x \log \log ^{4} x .
$$

Since $n(p) \leq g(p)$ this shows that on average the maximal value of $|A|$ cannot be greater than $\log ^{6} p$.

4. Lower bound. In this section we will show that the maximal value of $|A|$ is at least $\Omega(\log \log p)$. The proof is based on Weil's estimation of character sums.

THEOREM 4.1. There exists an $A \subset F_{p}$ such that $|A|=\Omega(\log \log p)$ and $\mathrm{FS}(A)$ avoids the set of quadratic nonresidues.

First we prove a lemma.

Lemma 4.2. Let $Q$ be the set of quadratic residues. Assume that for some set $B$ we have $Q+B=F_{p}$. Then $|B| \geq \frac{1}{4} \log p$.

Proof. Let $B=\left\{b_{1}, \ldots, b_{k}\right\}$ and $Q_{i}=Q+b_{i}$. Then

$$
\left|F_{p}-\bigcup_{i=1}^{k} Q_{i}\right|=\left|F_{p}\right|-\sum\left|Q_{i}\right|+\sum\left|Q_{i} \cap Q_{j}\right|-\cdots
$$

by the inclusion-exclusion formula. Now,

$$
\left|Q_{i_{1}} \cap \cdots \cap Q_{i_{l}}\right|=\sum_{a \in F_{p}} \frac{1}{2^{l}}\left(1+\left(\frac{a-b_{i_{1}}}{p}\right)\right) \cdots\left(1+\left(\frac{a-b_{i_{l}}}{p}\right)\right)+m\left(i_{1}, \ldots, i_{l}\right)
$$

where $\left|m\left(i_{1}, \ldots, i_{l}\right)\right| \leq l / 2$, since it may occur that $a-b_{i_{j}}=0$. By Weil's theorem [5],

$$
\left|\sum_{n=1}^{p}\left(\frac{f(n)}{p}\right)\right| \leq(t-1) \sqrt{p}
$$

where $f(x)=\prod_{i=1}^{t}\left(x-a_{i}\right)$ and $a_{1}, \ldots, a_{t}$ are distinct elements of $F_{p}$. Multiplying out the product we see that

$$
\left(1+\left(\frac{a-b_{i_{1}}}{p}\right)\right) \ldots\left(1+\left(\frac{a-b_{i_{l}}}{p}\right)\right)=1+\sum\left(\frac{f(a)}{p}\right)
$$

where $f$ runs through the $2^{l}-1$ polynomials of the type considered above. Hence

$$
\left|Q_{i_{1}} \cap \cdots \cap Q_{i_{l}}\right|=p / 2^{l}+m^{\prime}\left(i_{1}, \ldots, i_{l}\right)
$$

where $\left|m^{\prime}\left(i_{1}, \ldots, i_{l}\right)\right| \leq 2^{-l}\left(2^{l}-1\right)(l-1) \sqrt{p}+l / 2$. We can assume that $l \leq k \leq \sqrt{p}$ (if $k \geq \sqrt{p}$ we are done). Thus $\left|m^{\prime}\left(i_{1}, \ldots, i_{l}\right)\right| \leq k \sqrt{p}$. It follows 
that

$$
\begin{aligned}
0=\left|F_{p}-\bigcup_{i=1}^{k} Q_{i}\right| & =p-\sum_{i=1}^{k}\left(\frac{p}{2}+m^{\prime}(i)\right)+\sum\left(\frac{p}{4}+m^{\prime}(i, j)\right)-\cdots \\
& =p(1-1 / 2)^{k}+M
\end{aligned}
$$

where $|M| \leq 2^{k} k \sqrt{p}$. Hence $p / 2^{k}=|M| \leq 2^{k} k \sqrt{p}$, thus $\sqrt{p}<k 4^{k}<e^{2 k}$ so that $k \geq \frac{1}{4} \log p$.

REMARK 5. Clearly the same statement holds for the set $R$ of quadratic nonresidues.

Proof of Theorem 4.1. Let $A$ be a maximal set for which $\mathrm{FS}(A)$ avoids the quadratic nonresidues. We will show that

$$
|A| \geq \frac{1}{\log 2} \log \log p-2 .
$$

Suppose otherwise. Then $|\mathrm{FS}(A)| \leq 2^{|A|} \leq \frac{1}{4} \log p$, thus $R-\mathrm{FS}(A) \neq F_{p}$ so there exists an $s \in F_{p}$ for which $s \notin R-\left(a_{i_{1}}+\cdots+a_{i_{l}}\right)$ for any $a_{i_{1}}, \ldots, a_{i_{l}} \in A$. Hence one can add $s$ to $A$, which contradicts the maximality of $A$.

REMARK 6. There exists a set $B$ for which $|B|=[10 \log p]$ and $Q+B$ $=F_{p}$. Let us choose the elements of $B$ independently at random with probability $P(b \in B)=(c \log p) / p$. Then

$$
P(x \notin Q+B)=\prod_{i=1}^{(p-1) / 2} P\left(x-i^{2} \notin B\right)=\left(1-\frac{c \log p}{p}\right)^{(p-1) / 2}
$$

since we have chosen the elements independently. Hence

$$
P\left(Q+B \neq F_{p}\right) \leq \sum_{x=0}^{p-1} P(x \notin Q+B)=p\left(1-\frac{c \log p}{p}\right)^{(p-1) / 2} \leq p e^{-\frac{1}{3} c \log p} .
$$

On the other hand, by the Chernoff inequality [6] we have

$$
P(|| B|-c \log p| \geq \lambda \sigma) \leq 2 \max \left(e^{-\lambda^{2} / 4}, e^{-\lambda \sigma / 2}\right)
$$

where

$$
\frac{1}{2} c \log p \leq \sigma^{2}=p \frac{c \log p}{p}\left(1-\frac{c \log p}{p}\right) \leq c \log p .
$$

Choosing $c=4$ and $\lambda=\sqrt{8 \log p}$ we get

$$
P(|| B|-4 \log p| \geq 4 \sqrt{2} \log p) \leq 2 e^{-2 \log p}=2 / p^{2} .
$$

We have $p e^{-\frac{4}{3} \log p}=p^{-1 / 3}$. Since $2 / p^{2}+1 / p^{1 / 3}<1$ for $p \geq 3$, with positive probability we have $|B| \leq 10 \log p$ and $Q+B=F_{p}$.

We have shown that in the case $\left(\frac{2}{p}\right)=-1$ we have $|\operatorname{FS}(A)|=2^{|A|}$. Thus in general probably one cannot get an estimate better than $\Omega(\log \log p)$, 
since after the selection of $|A|-1$ elements the set of subset sums has $2^{|A|-1}$ elements and it cannot be the additive complement of $-R$, while sets with more than $10 \log p$ elements are such complements with high probability.

Acknowledgements. I profited much from discussions with A. Sárközy and K. Gyarmati.

\section{References}

[1] D. A. Burgess, The distribution of quadratic residues and non-residues, Mathematika 4 (1957), 106-112.

[2] D. A. Burgess and P. D. T. A. Elliott, The average of the least primitive root, ibid. 15 (1968), 39-50.

[3] P. Erdős and A. Sárközy, Arithmetic progressions in subset sums, Discrete Math. 102 (1992), 249-264.

[4] P. X. Gallagher, A larger sieve, Acta Arith. 18 (1971), 77-81.

[5] W. E. Schmidt, Equations over Finite Fields. An Elementary Approach, Springer, Berlin, 1975.

[6] T. Tao and V. Vu, Additive Combinatorics, Cambridge Stud. Adv. Math. 105, Cambridge Univ. Press, Cambridge, 2006 (p. 24).

Department of Algebra and Number Theory

Eötvös Loránd University

Pázmány Péter sétány 1/C

H-1117 Budapest, Hungary

E-mail: csiki@cs.elte.hu

Received on 25.10.2007

and in revised form on 29.6.2008 